\title{
A trade-off between Energy Efficiency and Spectral Efficiency in macro-femtocell networks
}

This paper was downloaded from TechRxiv (https://www.techrxiv.org).

\section{LICENSE}

CC BY 4.0

SUBMISSION DATE / POSTED DATE

09-02-2021 / 10-02-2021

\section{CITATION}

Ghosh, Joydev (2021): A trade-off between Energy Efficiency and Spectral Efficiency in macro-femtocell networks. TechRxiv. Preprint. https://doi.org/10.36227/techrxiv.13783036

$\mathrm{DOI}$

10.36227/techrxiv.13783036 


\title{
A trade-off between Energy Efficiency and Spectral Efficiency in macro-femtocell networks
}

\author{
Joydev Ghosh, Member, IEEE
}

\begin{abstract}
Obtaining large spectral efficiency (SE) and energy efficiency (EE) subject to quality of experience (QoE) is one of the prime concerns for the wireless next generation networks, however a major confrontation with its trade-off which is becoming apparent while optimizing both $\mathrm{SE}$ and $\mathrm{EE}$ parameters concurrently. In this work, an analytical framework for a cognitive-femtocell network is proposed to be dealt with and overcome the situations regarded as unwelcome. Here, the conflict of SE-EE trade-off in downlink (DL) transmission is expressed methodically by Pareto Optimal Set (POS) based on a multi-empirical most effective use of a resource scheme as a function of femto base station (FBS) and macro base station (MBS) transmit power and base station (BS) density, respectively. Then, SE and EE are formulated in a utility function by applying Cobb-Douglas production function to transform the multi-empirical difficulty into the single-empirical optimization case. Besides, it is analytically shown that the SE-EE trade-of can be optimize through a distinctive universal optimum among the Pareto optimal by fine-tuning the weighting metric other than BS transmit power and density, respectively. Simulation results validate that it is possible to obtain the EE-SE trade-off with SINR threshold at different weighting factor.
\end{abstract}

Index Terms-Macrocell, Femtocell, Utility Function, Pareto Optimal Set, Energy Efficiency, Spectral Efficiency

\section{INTRODUCTION}

Nowadays heterogeneous cellular networks (HetNets) in wireless communication compose of macro base stations (MBSs), where each MBS is overspread with number of femto base stations (FBSs). In [1], the cognitive-femtocell network scenario consisting of macro base station (MBSs) positioned in a circular grid shape whilst the femtocells are indiscriminately located within the network coverage of each MBS. Provided this network framework, presented in [2], an optimal FBS number can possible achieve with respect to a macrocell which optimizes the efficiency level of the network performance at different traffic. From the survey of the optimal FBS density in order to restrict the outage probability, this is demonstrated in [3], [4] that different functional structure possible to establish in the interference restricted system which permits the EE accomplishment to be optimized. The work presented in [5]-[7] illustrated the viability of obtaining the remarkable furtherance in the SE response of a two-tier network.

At a recent time, a literature review dealing with nearly all elements of the deployment and functional methods for

Joydev Ghosh is with School of Computer Science and Robotics, National Research Tomsk Polytechnic University, RUSSIA, Email: joydev.ghosh.ece@gmail.com. improving the SE and EE response is given in [12]. Power tuning alongside orthogonal spectrum allotment can be a propitious radio resource management (RRM) strategy to attenuate co-channel interference in the system level keeping large EE response [8], [9]. The fruitfulness of attenuating the unwanted signal with optimal power tuning (OPT) method is probed in [10], [11], [13] where it is demonstrated that the accomplishment obtained at the receiver can be improved by making unwanted signal power lower than a specific limit and putting an extra attention on the importance of making use of power tuning at the transmitter. The EE response alongside the maximal OPT metrics for optimizing the EE are computed in [19] applying a model that describes the structure of cell membranes for estimating the interference achieved at a precise cell of interest presuming that the unwanted FBSs are positioned exterior to that of the macrocell network coverage circles of constant radius.

For a widely applicable system model configuration comprising of UE, MBSs and FBSs which are deployed as two independent spatial Poisson point process (PPP) distribution, the presented work of [15] determines the obtainable coverage probability formulation in the closed form expression applying the Stochastic Geometry Tools. Even though the discovery in [16]-[18] exhibit promising outcomes for enhancing the SE applying OPT, the emphasis of these surveys is limited to the uplink (UL) transmission gives only insufficient comprehension on the comprehensive network response. Furthermore, the potential to procure less energy consumptions at FBSs or MBSs through fine-tune the power in DL transmission not been discussed in detail in [10]. At a recent time, the efficacy of performance on the capacity whilst experiencing the smallest amount of power dissipation in DL at the FBSs is presented in [22], [24] where the power attenuations obtained are an outcome of executing a adaptive power tune method at the FBSs. The SE response of a two-layer wireless network with the DL OPT approach is probed in [20], [21], [25] applying Stochastic Geometry Tools where outstanding performance are exhibited by adjusting the FBS transmit power depending upon path loss between a FBS and an UE.

\section{A. Background and Related Work}

Based on Shannon's law, conflicts of objects may occur while optimizing both $\mathrm{EE}$ and SE simultaneously but it is necessary to optimizing both of them simultaneously in an ideal case, therefore a trade-off lies between two parameters [36]. Moreover, optimizing either EE or SE does not signify that another one is also becoming optimized. As a matter 
of fact, the optimum EE response may result in bad SE response and vice verse. Thus, it is utmost concerned in cellular networks to establishing a trade-off between $\mathrm{EE}$ and SE. The EE-SE trade-off has been demonstrated in [37], [38] for circular array antenna at each node putting the special attention by eliminating out-of-cell interference in the highrate reliable vehicular communications and also to the places where both transmitters and receivers having whole channel state information.

Existing literatures investigations on EE-SE trade-off can be classified into two types [39], [40]: first one is to articulating on the distinguished features between EE and SE as precisely as possible and second one is to optimize EE with an SE need. Particularly, in [41] and [42], the closed-form approximation for EE-SE trade-off has been demonstrated for numerous network models. In [43], [50], EE-SE trade-off maximization issue in orthogonal frequency division multiple access (OFDMA) based system model in DL transmission is presented to optimize EE with a minimum SE need. Besides, this optimization issue in DL distributed antenna networks is probed in [44], [49] to optimize EE by adjusting SE at a fixed level. Quite a few disadvantages can be identified in the preceding works. In the first type, because of deficiency of a distinctive solution in comprehensive optimization, the EE-SE trade-off unable to provide straight direction to the network operator; whereas in the second type, EE is optimized by limiting the SE performance.

To succeed in dealing with the disadvantages of preceding design foundation, a unite EE-SE trade-off metric has been proposed that may be applied to maximizing both EE and SE concurrently. The POS is the set of entire Pareto optimal nodes. Also, a node is Pareto optimal if and only if the rest of the points are unable make both of them (i.e., EE and SE) improved concurrently. To find the solution of a multiempirical difficulty, the distinctive universal optimal solution to be determined by making POS distinctive. The aim is to obtain a distinctive universal optimal solution even though entire nodes in $P^{P O S}$ is Pareto optimal. An effective technique to differentiate a distinctive node in POS is the Cobb-Douglas production technique. The multi-empirical optimization is a field of more than one benchmark resolution which is related to the mathematical optimization issues and integral part of more than one objective function to be optimized concurrently. Multi-empirical optimization may be useful in different areas of science and engineering where optimal verdicts require to be considered in the existence of trade-offs between the objects for their conflicts of interest. In [45]-[47], a multiempirical optimization technique is introduced to fix the resource allotment issue for EE-SE tradeoff. Furthermore, the multi-empirical optimization difficulty is shown to be similar to a single-empirical optimization difficulty in optimizing the obtainable data rate with limitation of less power consumption. To determine a distinctive universal optimal solution, the Cobb-Douglas production is applied to transform multiempirical difficulty into the single-empirical optimization case [39], [48].

\section{B. Contributions and Organization}

The significant threefold contributions of this work that fulfill the paramount aim are outlined as follows:

- Initially the paper develops the closed-form expressions of network SE and network EE, respectively, for a cognitive-femtocell network and then examine methodically in detail in order to explain and interpret the insight of the various operational regimes and also to determine the feasible conditions for which optimum network performance can achieve.

- An analytical model development of a basic structure underlying a two-layer network to investigate and probe the different features of SE and EE in comparison to that of one layer network presented in [6]. The closed-form expressions as a function of transmit power, BS density, and SINR thresholds are explicitly demonstrated for SE and EE keeping the constraint that the probability of the indiscriminately distributed users reach a throughput level that cross the minimum threshold for QoE.

- To measure the amount of performance gap of SE to that of EE, the fundamentals of Pareto Optimal Set and CobbDouglas production function are utilized to expressing a new utility function to present the SE-EE tradeoff [40]. The use of EE-SE trade-off multi-empirical case is expressed mathematically in most effective way in (10) with the establishment of transmit power POS for constant BS density and the BS density POS for constant transmit power, respectively. Next, multi-empirical case is converted into the single-empirical optimization case by utility function and extends (10) to (19). Thereafter, it is proved that $\log _{e} U$ is strictly quasi-concave with regard to $p^{P O S}$ for fixed $\mathrm{BS}$ density and with regard to $\rho^{P O S}$ for constant transmit power respectively, proposed in Theorem 3 and Theorem 4. The graphical response of the SE-EE trade-off parameter is illustrated with regard to different weighting factor and also at different optimal transmit power to probing the potential merits of SE and EE to get them balance for the introduced network.

The remainder of this paper is outlined as follows. Section II describes the system model. Section III is the most important technical part of this work, where the expressions of major parameters are presented for the system model. Furthermore, numerical and simulation results are analyzed and discussed in Section IV before the conclusion in Section V. Finally, proofs are provided in the Appendix to increase the readability.

\section{System MOdEL}

The down link (DL) system model is regulated by the considerations listed over the succeeding sub-sections.

- Network architecture: A double-layer cellular network in which deployment of a large number of Femto cells is been put into effect within the network of macrocell. The users (i.e., known as underprivileged users) located far apart from the MBS will get service by the cognitivefemtocell network which in turn aids to provide consistent quality of experience (QoE). The FBS access the assigned 
frequency band utilizing the OFDMA [23] technique. Here, each FBS can concurrently maintain DL communication to other users within its vicinity.

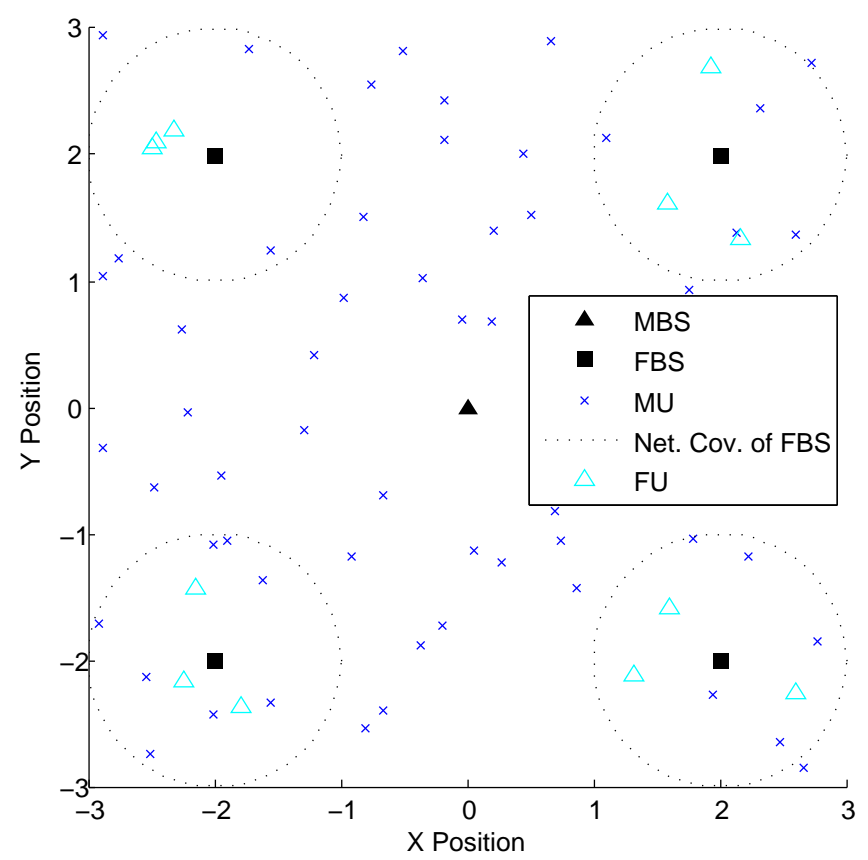

Fig. 1: A two-tier heterogeneous cellular network model consists of macrocell and femtocell

- FBS and MBS deployment: Two independent spatial Poisson point process (PPP) distribution, and FBS and MBS density per unit area as $\rho_{f}$ and $\rho_{m}$ respectively are taken into account for the deployment of the set of FBSs (i.e., $\Psi_{f}$ ) and the deployment of the set of FBSs (i.e., $\Psi_{m}$ ) in the network model. The position of a FBS and a MBS can be denoted by $\mathbf{F}_{\mathbf{i}}$ and $\mathbf{M}_{\mathbf{g}}$ for its index $i \in \Psi_{f}$ and $g \in \Psi_{m}$.

- User distribution: In Fig. 1, we considered that users are randomly distributed, and $\mathbf{S}_{\mathbf{i}}$ be the set of users in the Femto cells and $\mathbf{S}_{\mathrm{g}}$ be the set of users in the Macro cells. Network coverage radius of a femto cell can be denoted by $r$. Assume the FU of interest as FU $s \in \mathbf{S}_{\mathbf{i}}$ and its position is indicated by $x_{i, s}$ for that $\left\|\mathbf{F}_{\mathbf{i}}-x_{i, s}\right\|=r_{i, s}$, where $r_{i, s} \leq r$ and $r_{i, s}$ represents the radial separation between FU $s$ and $i^{\text {th }}$ FBS, and $\left\|\mathbf{F}_{\mathbf{i}}-x_{i, s}\right\|$ represents the straight-line distance between two points $\mathbf{F}_{\mathbf{i}}$ and $x_{i, s}$ in Euclidean space. It is also considered that equal number of FUs is present there in each Femto cell without mislaying of generality, i.e., $\mathbf{S}_{\mathbf{i}}=S_{i}, \forall i \in \Psi_{f}$, where $S_{i}$ indicates number of elements in set $\mathbf{S}_{\mathbf{i}}$. Likewise, $\left|\mathbf{S}_{\mathbf{g}}\right|=S_{g}, \forall i \in \Psi_{m}$, where $S_{g}$ indicates number of elements in set $\mathbf{S}_{\mathbf{g}}$.

- Spectrum Allotment: The entire accessible spectrum, i.e., $B \mathrm{~Hz}$ is split up into $K$ number of radio resource blocks (RBs) which comprises of a set of orthogonal frequency, each of them utilizing an accessible spectrum of $b \mathrm{~Hz}$ in the context of the considered OFDMA. A set of successive RBs is merged to form an resource block group (RBG), i.e., $w_{s} \in W$, subject to no overlapping among RBs and RBGs (i.e., $w_{s} \cap w_{j}$, where $\{s, j\} \in \mathbf{S}_{\mathbf{i}}$, and $s \neq j$ ) to avoid intra-femtocell interference, where $w_{s}$ indicates set of RBs from RBG allocated to $s^{t h}$ FU. Additionally, to revamp SE of the network, it is considered that each FBS has the opportunity to use all the RBs. The RBs are completely exploited whilst transmitting to S FUs within the Femto cell. Furthermore, all FUs in a Femto cell are assigned with equal number of RBs (i.e., $\left|w_{s}\right|=w, \forall s \in \mathbf{S}_{\mathbf{i}}$ ) occupying an accessible spectrum of $w b \mathrm{~Hz}$ per FU.

- Transmit power: It is considered that all FBSs (i.e., $\Psi_{f}$ ) transmit accounting uniform power (i.e., $p_{f} \mathrm{~W}$ ) per RBG. Therefore, total transmitted power for $S$ number of FUs (i.e., $P=S_{i} p_{f}$ ) by a FBS is assigned over the complete RBGs.

- Channel Model: The channel model is consist of the large scale path loss (LSPL) and the small scale fading (SSF), where small scale fading is superimposed over additive white Gaussian noise (AWGN) channel and average thermal noise power, $\tau_{0}$, is considered to characterized AWGN channel.

On the whole, the advantages of the proposed model are listed as below:

1) It is worthy for short distance and long distance communication, respectively and also this gives the better performance evaluation in contrast to the other channel models.

2) The capability to obtain comparatively plain expressions and manifest explanatory comprehensions on the system model accomplishment, mainly in the restriction of a multilayer networks co-existence scenario.

3) A model of remarkable versatility as it can allow fine-tune the parameters such as weighting factor and SINR, respectively, to apprehend different propagation characteristics.

4) The physical interpretation for some values of weighting factor has been investigated in the section III.

5) The practicality of this model is suitable for the implementation as it is bounded and continuous.

In spite of the above, the number of shortcomings can also be identified. The proposed model is restricted to a two-tier model of the heterogeneous cellular networks, although it can extend for even more tiers but for that it is recommended to incorporate well established path loss models as special case. Thus, neither it can be claimed that the versatility and practicality of the system model is the best network model nor that it can take the place of existing heterogeneous network models. Preferably, it is an another proposed model which is appropriate for vehicular communications and it permits us to demonstrate interesting findings that could be perceived in practice.

\section{Performance AnAlysis}

\section{A. DL Success Probability:}

The success probability (SP), i.e., $\eta$, can be defined as the probability that the SINR of the FU during the signal 
transmission of its associated FBS higher than the required set SINR threshold, i.e., $\varphi$. Therefore, based on the considerations 1-6 in section II, the SINR by the FU of interest $s$ during the signal transmission of its associated FBS $i$ can be expressed by:

$$
\gamma_{i, s}=\frac{p_{f} h_{i, s} d_{i, s}^{-\alpha}}{\sum_{j \in \Psi_{f}} p_{f} h_{j, s} d_{j, s}^{-\alpha}+\sum_{g \in \Psi_{m}} p_{m} h_{g, s} d_{g, s}^{-\alpha}+\tau_{0}},
$$

where $h_{i, s}, h_{j, s}$ and $h_{g, s}$ represent SSF coefficient of the channel between $i^{\text {th }}$ FBS and $s^{\text {th }}$ FU, between interfering $j^{\text {th }} \mathrm{FBS}$ and $s^{\text {th }} \mathrm{FU}$, and between $g^{\text {th }}$ MBS and $s^{t h} \mathrm{FU}$ respectively; $d_{i, s}^{-\alpha}, d_{j, s}^{-\alpha}$ and $d_{g, s}^{-\alpha}$ represent LSPL between $i^{t h}$ FBS and $s^{t h}$ FU, between interfering $j^{t h}$ FBS and $s^{t h}$ FU, and between interfering $g^{\text {th }}$ MBS and $s^{\text {th }}$ FU respectively, where the path loss exponent $\alpha>2 ; I_{f}=\sum_{j \in \Psi_{f}} p_{f} h_{j, s} d_{j, s}^{-\alpha}$ indicates co-tier interference generated due to other FBSs (i.e. $j \neq i$ ) for communicating on the same RBG allotted to $s^{t h}$ FU, $I_{m}=\sum_{g \in \Psi_{m}} p_{m} h_{g, s} d_{g, s}^{-\alpha}$ indicates cross-tier interference generated due to the MBSs for communicating on the same RBG allotted to $s^{\text {th }} \mathrm{FU}$ and $\tau_{0}$ stands for average thermal noise power.

For the assumption of the Homogeneous Poisson Point Process (H-PPP) system model, the probability that $i^{\text {th }}$ FBS at $\mathbf{F}_{\mathbf{i}}$ can successfully transmit to $s^{\text {th }}$ FU located at the origin (i.e., $x_{i, s}=0$ ) as $\eta\left(d, \rho_{f}, \Psi_{f}\right)=\mathbf{P}^{\mathbf{0}}\left[\gamma_{i, s}>\Psi_{f}\right]$, where $\mathbf{P}^{\mathbf{0}}$ and $\rho_{f}$ represent Palm distribution and density of FBSs deployment per $m^{2}$, respectively. According to the Slivnyaks theorem, $\eta$ is identical for other FBSs for their transmission to the FUs. Following the method provided in [26], $\eta$ is expressed as (proof is delegated in Appendix A):

$$
\eta\left(d, \rho_{f}, \Psi_{f}\right)=\exp \left[-\rho_{f} \xi_{\alpha}-\frac{\zeta}{p_{f}}\right]
$$

where $\xi_{\alpha}=d^{2} \Psi_{f}^{\frac{2}{\alpha} \lambda_{\alpha}}$ is the FBS coverage influential metric, where $\lambda_{\alpha}=2 \pi \Gamma\left(\frac{2}{\alpha}\right) \Gamma\left(1-\frac{2}{\alpha}\right) \alpha^{-1}$ (where $\Gamma(m)$ stands for the Gamma function of $m$ ) is a parameter that dependent on pathloss exponent; $\zeta=\varepsilon \Psi_{f} d^{\alpha} \tau_{0}$ is a metric that dependent on channel condition and $\Psi$.

\section{B. Network Spectral Efficiency:}

At each interval of time, the density of FBSs (i.e., $\rho_{f}$ ) communicating in the DL on an RBG is a random variable. Additionally, the SP is also dependent on $\rho_{f}$ each interval of time, as stipulated in (2). Although, by averaging on large iterations, the possibility of transmitting signal successfully can be similar for entire FBS to FU pairs in the H-PPP system model according to the Slivnyaks theorem. The SE can be re-expressed with the incorporation of density of FBSs (i.e., $\rho_{f}$ ) and MBS (i.e., $\rho_{m}$ ), as first given in [6], based on the involvement in DL concurrent transmission :

$$
\begin{aligned}
\phi_{S E}\left(\rho_{f}, \rho_{m}\right) & =\rho_{f} S_{i} \exp \left[-\rho_{f} \xi_{\alpha}-\frac{\zeta}{p_{f}}\right] \frac{w b}{B} \log _{2}\left(1+\varphi_{f}\right) \\
& +\rho_{m} S_{g} \exp \left[-\rho_{m} \xi_{\alpha}-\frac{\zeta}{p_{m}}\right] \frac{w b}{B} \log _{2}\left(1+\varphi_{m}\right) \\
& =\rho_{f} \exp \left[-\rho_{f} \xi_{\alpha}-\frac{\zeta}{p_{f}}\right] \log _{2}\left(1+\varphi_{f}\right) \\
& +\rho_{m} \exp \left[-\rho_{m} \xi_{\alpha}-\frac{\zeta}{p_{m}}\right] \log _{2}\left(1+\varphi_{m}\right),
\end{aligned}
$$

The SE established in (3) signifies the degree of compactness of transmission in the system model, which is estimated by the multiplication of density of successful transmission to the throughput per unit bandwidth (BW). In this regard, the throughput per unit BW obtained by the transmission of only one FBS on an RBG with BW of $w b \mathrm{~Hz}$ is expressed as the multiplication of $\eta\left(d, \rho_{f}, \Psi_{f}\right)$ and $\frac{w b}{B} \log _{2}\left(1+\varphi_{f}\right)$. Therefore, throughput of a Femto cell is obtained by the product of $\frac{w b}{B} \log _{2}\left(1+\varphi_{f}\right)$ and $S_{i}$, where $S_{i}$ indicates number of FUs in a Femto cell and enhancement in the quantity of the $\mathrm{SE}$ is then achieved by the incorporation of $\rho_{f}$. Thus, (3) is explicated as the system SE. It is deduced from (3) that enhancing FBS density causes a superior SE, nevertheless, it is to some extent as described in the succeeding. Instinctively, a large FBS density causes an interference-limited process, where simultaneous transmissions by interfering FBSs worsen the SP on all links cause lesser SE. The response of SE is characterized by

\section{Theorem 1.}

Theorem 1: The SE is strictly quasi-concave in $\left\{\rho_{f}, \rho_{m}\right\} \in\{0, \infty\}$. Hence, the universal optimal SE is distinctive.

Proof: Proof is delegated in Appendix B.

Subjected to Theorem 1, the optimum BS density which optimizes the SE without any outage limitation can be indicated by $\rho_{S E}^{*}$ and this can be achieved by configuring to zero the derivative of $\phi_{S E}\left(\rho_{f}\right)$ with respect to $\rho_{f}$ (i.e., $\left.\frac{\phi_{S E}\left(\rho_{f}\right)}{\rho_{f}}\right)$. Hence, we can obtain $\rho_{S E}^{*}=\frac{1}{\xi_{\alpha}}$ by settling for $\rho \stackrel{\rho_{f}}{=} \rho_{S E}^{*}$. Therefore, the optimum SE obtained at $\rho_{S E}^{*}$ under no outage limitation is approximately expressed by $\log _{2}\left(1+\varphi_{f}\right) \frac{e^{-1}}{\xi_{\alpha}}$, which is followed for interference limited condition whilst $\rho_{f} \xi_{\alpha}>>\frac{\zeta}{p_{f}}$. This can be ensured that the success probability of $e^{-1} \approx 0.37$ at $\rho_{S E}^{*}$ is unsatisfactory for the operation of conventional network under no outage limitation. Thus, the SE can be maximized by incorporating an outage probability (OP) entity $k$ corresponding to $\varphi$ as:

$$
\begin{aligned}
& \max _{p_{f}} \phi_{S E}\left(\rho_{f}, \rho_{m}\right) \text { Subject to: } 1-\eta\left(d, \rho_{f}, \Psi_{f}\right) \leq k, \\
& \text { and } \max _{p_{m}} \phi_{S E}\left(\rho_{f}, \rho_{m}\right) \text { Subject to: } 1-\eta\left(d, \rho_{m}, \Psi_{m}\right) \leq k,
\end{aligned}
$$

where $1-\eta\left(d, \rho_{f}, \Psi_{f}\right)$, positioned on the left of the above inequality, indicates the OP. The solution of (4) achieved 
by the Lagrange function can be expressed as $\mathcal{L}\left(\rho_{f}, \lambda\right)=$ $\phi_{S E}\left(\rho_{f}\right)-\lambda\left[1-\eta\left(d, \rho_{f}, \Psi_{f}\right)-k\right]$, where the $\lambda$ term stands for Lagrange multiplier. For the condition $k\left\langle 1-e^{-1}, \lambda\right\rangle$ 0 and this implies that the OP constraint is enabled i.e., $1-\eta\left(d, \rho_{f}, \Psi_{f}\right)=k$, followed by the optimum network BS density, i.e., $\rho=\left(\rho_{f}+\rho_{m}\right)$, is achieved as:

$\rho^{*}=\frac{\left\{-\log _{2}\left(1-k_{f}\right)-\zeta p_{f}^{-1}\right\}+\left\{-\log _{2}\left(1-k_{m}\right)-\zeta p_{m}^{-1}\right\}}{\xi_{\alpha}}$,

where $\rho^{*}$ aids to optimise the SE under an outage limitation. As $\eta$ (ref. to [2]) is a function which exponentially decreases with $\rho$, the optimum FBS density (i.e., $\rho^{*}$ ) reposes at a point under an outage limitation is all the time smaller than $\rho_{S E}^{*}$ for $\left\{k_{f}, k_{m}\right\}<0.4$ whilst OP entity grown to $k_{f}=1-e^{-1-\frac{\zeta}{p_{f}}}$ and $k_{m}=1-e^{-1-\frac{\zeta}{p_{m}}}$ for the user taking the service from Femtocell or Macrocell respectively and it is possible to obtain a BS density that optimises under an outage limitation (see an outage-limitation SE) at an alike BS density that optimises the SE under no outage limitation i.e., $\rho^{*}=\rho_{S E}^{*}$. Obviously, this is not achievable as this framework matches almost exactly to a subject of study whilst outage entity is $\left\{k_{f}, k_{m}\right\}>1-e^{-1} \approx$ 0.63 . Hence including the numerical value of $k$, the highest outage limitation SE reached at $\rho^{*}$ can become:

$\phi_{S E}\left(\rho^{*}\right)=\frac{\begin{array}{c}\left(1-k_{f}\right)-\log _{2}\left(1-k_{f}\right)-\zeta p_{f}^{-1} \log _{2}\left(1+\varphi_{f}\right) \\ +\left(1-k_{m}\right)-\log _{2}\left(1-k_{m}\right)-\zeta p_{m}^{-1} \log _{2}\left(1+\varphi_{m}\right)\end{array}}{\xi_{\alpha}}$.

From the above equation, it can be noticed that $\phi_{S E}\left(\rho^{*}\right)$ is also highly influenced by $\varphi_{f}$ and $\varphi_{m}$. In this case, fixing $\varphi_{f}$ and $\varphi_{m}$ to a high value will normally indicate that the $\eta$ will be even lesser whilst working in a large interference situation. Thus, to retain a specific SE specified outage object, the amount of interference in the system model should be regulated by restricting $\rho_{f}$ and $\rho_{m}$ that exploit the allocated frequency band. In noise-limited scenarios, enhancing the transmit power level of FBS and MBS up to the maximum possible transmit power, i.e., $P_{f}$ and $P_{m}$, permit to enhance the SE. Although interference limited scenarios, the utilization of large transmit power cannot enhance the SE due to associated grow in the received signal strength (RSS) cancel each other out by large interference power from the adjacent BSs. As the difficulty is to exploit the network operation in allocated spectrum with interference limited scenarios, we neglect $\tau_{0}$ by taking both $\rho_{f} \xi_{\alpha}>>\frac{\zeta}{p_{f}}$ and $\rho_{m} \xi_{\alpha}>>\frac{\zeta}{p_{m}}$ into account. Point to be noted that this will not have an impact on the high SE attainments because of less effect of $\zeta$ on $\eta$. Based on this consideration, the SINR threshold $\varphi_{f}$ and $\varphi_{m}$ can be reconsidered by the SIR threshold $\varphi_{f}^{\prime}$ (i.e., $\varphi_{f} \approx \varphi_{f}^{\prime}$ ) and $\varphi_{m}^{\prime}$ (i.e., $\varphi_{m} \approx \varphi_{m}^{\prime}$ ).

\section{Network Energy Efficiency:}

Following the power dissipation model as given in [6], the EE can be established by defining it as the total throughput obtained over an allocated RBG with BW $B$, which is normalized by the sum power dissipation in the system model.

$$
\phi_{E E}\left(\rho_{f}, \rho_{m}\right)=\frac{\begin{array}{c}
\rho_{f} \exp \left(-\rho_{f} \xi_{\alpha}-\frac{\zeta_{\alpha}}{p_{f}}\right) B \log _{2}\left(1+\varphi_{f}\right) \\
+\rho_{m} \exp \left(-\rho_{m} \xi_{\alpha}-\frac{\zeta_{\alpha}}{p_{m}}\right) B \log _{2}\left(1+\varphi_{m}\right)
\end{array}}{\rho_{f} Q_{f}+\rho_{m} Q_{m}}
$$

where power dissipation at an individual BS in the femto and macro layers can be expressed as:

$$
\left.\begin{array}{l}
Q_{f}=a_{f} P_{f}+b_{f} \\
Q_{m}=a_{m} P_{m}+b_{m}
\end{array}\right\},
$$

where $a_{f}$ and $a_{m}$ indicate coefficient of a BS belongs to the femto or macro layer respectively. Here, $P_{f}$ and $P_{m}$ represent the total transmits power assigned to $S_{i}$ and $S_{g}$ users over the complete RBGs is given by $P_{f}=S_{i} p_{f}$ and $P_{m}=S_{g} p_{m}$ respectively. $b_{f}$ and $b_{m}$ denote power-offset which compensate FBS and MBS power for added attenuation and amplification during the signal transmission.

Theorem 2: The EE is strictly quasi-concave in $\rho_{f} \in$ $\{0, \infty\}, \forall \rho_{m}, \varphi_{f}, \varphi_{m}, \zeta, \xi_{\alpha}, p_{f}, p_{m}, Q_{f}, Q_{m}>0$. Hence, universal optimum femto-tier BS density $\rho_{f}^{u}$ that makes the outage non-restricted $\mathrm{EE}$ as large as possible and its solution can be given by:

$$
\exp \left(\rho_{f}^{u}, z, \zeta\right)=\mu \frac{\rho_{m} Q_{m}-\rho_{f}^{u} \xi_{\alpha}\left(\rho_{m} Q_{m}+\rho_{f}^{u} Q_{f}\right)}{\left(Q_{f}+\in \zeta\left(\rho_{m} Q_{m}+\rho_{f}^{u} Q_{f}\right)\right.},
$$

where $\mu=\frac{\log _{2}\left(1+\varphi_{f}\right) \exp \left(\rho_{m}, L\right)}{\rho_{m} \log _{2}\left(1+\varphi_{m}\right)}$ and $L=\xi_{\alpha}\left(p_{f}^{-1}-1\right)$ Proof: Proof is delegated in Appendix C.

The conclusion that can be drawn from the quasi-concave characteristic of EE for the dual-layer is that, for a network arrangement as stated in Theorem 2, growing $\rho_{f}$ further results in a quick grow in the SE than the total power absorbed by the two tiers. This context, defined by the region $\rho_{f} \in\left[0, \rho_{f}^{u}\right]$, is categorized as the growing energy efficiency (G-EE) pattern. Above $\rho_{f}^{u}$, the total power absorbed by the two tiers of the networks overloads on the obtained SE, resulting in a reducing energy efficiency (R-EE) pattern where the EE response reduces from its maximum value as $\rho_{f}$ grows. It may follow to a point where the networks EE is equal to that obtained by the only macro-tier.

\section{Spectral Efficiency-Energy Efficiency Trade-off:}

A multi-empirical most effective use of a resource scheme is proposed to deal with the resource allocation difficulty for EESE trade-off that can be adjustable based on the importance of EE or SE function by applying a tradeoff metric. The most effective use of EE-SE trade-off multi-empirical case is presented as follows: 
$C 1: \max _{p_{f}, p_{m}, \rho_{f}, \rho_{m}} \phi_{S E}\left(p_{f}, p_{m}, \rho_{f}, \rho_{m}\right), \phi_{E E}\left(p_{f}, p_{m}, \rho_{f}, \rho_{m}\right)$, Subject to: $\left\{p_{f}, p_{m}\right\} \in \mathbf{p},\left\{\rho_{f}, \rho_{m}\right\} \in \rho$

where $\mathbf{p}=\left[0 \leq\left\{p_{f}, p_{m}\right\} \leq\left\{p_{f}^{\max }, p_{m}^{\max }\right\}\right]$ and $\rho=$ $\left[1 \leq\left\{\rho_{f}, \rho_{m}\right\} \leq\left\{\rho_{f}^{\max }, \rho_{m}^{\max }\right\}\right]$ are the transmit power limitation and the set of selected FBS and MBS density, respectively.

An allocation $\bar{z}^{*} \in Z$ can be Pareto Optimal Set (POS) if there is no other feasible allocation $\bar{z} \in Z$ satisfying the condition $f_{i}(\bar{z}) \geq f_{i}\left(\bar{z}^{*}\right), \forall i=\{1,2, \cdots n\}$, where $f_{i}(z)$ is the empirical function [27], [28], [35].

Particularly, here, $\left\{p_{f}, p_{m}\right\} \in p$, or $\left\{\rho_{f}, \rho_{m}\right\} \in \rho$ is POS if there is no other feasible $p_{f}^{\prime}$ or $p_{m}^{\prime}$ or $\rho_{f}^{\prime}$ or $\rho_{m}^{\prime}$ that satisfies $\phi_{S E}\left(p_{f}^{\prime}, p_{m}^{\prime}\right) \geq \phi_{S E}\left(p_{f}, p_{m}\right)$ and $\phi_{E E}\left(p_{f}^{\prime}, p_{m}^{\prime}\right) \geq \phi_{E E}\left(p_{f}, p_{m}\right)$ or $\phi_{S E}\left(\rho_{f}^{\prime}, \rho_{m}^{\prime}\right) \geq$ $\phi_{S E}\left(\rho_{f}, \rho_{m}\right)$ and $\phi_{E E}\left(\rho_{f}^{\prime}, \rho_{m}^{\prime}\right) \geq \phi_{E E}\left(\rho_{f}, \rho_{m}\right)$.

Corollary 1: For constant $\left\{\rho_{f}, \rho_{m}\right\}$, the POS of transmit power for case $(C 1)$ is:

$$
\mathbf{p}^{P O S}=\left\{\begin{array}{ll}
\left\{p_{f} \mid p_{f}=p_{f}^{\max }\right\} ; & p_{f}^{*} \geq p_{f}^{\max } \\
\left\{p_{f} \mid p_{f}^{*} \leq p_{f} \leq p_{f}^{\max }\right\} ; & p_{f}^{*} \leq p_{f}^{\max }
\end{array} .\right.
$$

Similarly,

$$
\mathbf{p}^{P O S}= \begin{cases}\left\{p_{m} \mid p_{m}=p_{m}^{\max }\right\} ; & p_{m}^{*} \geq p_{m}^{\max } \\ \left\{p_{m} \mid p_{m}^{*} \leq p_{m} \leq p_{m}^{\max }\right\} ; & p_{m}^{*} \leq p_{m}^{\max }\end{cases}
$$

where $p_{f}^{*}$ and $p_{m}^{*}$ indicate the existence of the universal optimal to maximize $\phi_{S E}\left(p_{f}, p_{m}\right)$ and $\phi_{E E}\left(p_{f}, p_{m}\right)$.

Corollary 2: For constant $\left\{p_{f}, p_{m}\right\}$, the POS of transmit power for case $(C 1)$ is:

$$
\rho^{P O S}= \begin{cases}\left\{\rho_{f} \mid \rho_{f}=\rho_{f}^{\max }\right\} ; & \rho_{f}^{*} \geq \rho_{f}^{\max } \\ \left\{\rho_{f} \mid \rho_{f}^{*} \leq \rho_{f} \leq \rho_{f}^{\max }\right\} ; & \rho_{f}^{*} \leq \rho_{f}^{\max }\end{cases}
$$

Similarly,

$$
\rho^{P O S}= \begin{cases}\left\{\rho_{m} \mid \rho_{m}=\rho_{m}^{\max }\right\} ; & \rho_{m}^{*} \geq \rho_{m}^{\max } \\ \left\{\rho_{m} \mid \rho_{m}^{*} \leq \rho_{m} \leq \rho_{m}^{\max }\right\} ; & \rho_{m}^{*} \leq \rho_{m}^{\max },\end{cases}
$$

where $\rho_{f}^{*}$ and $\rho_{m}^{*}$ indicate the existence of the universal optimal to maximize $\phi_{S E}\left(p_{f}, p_{m}\right)$.

Based on Corollary 1 and Corollary 2, two problems can be separated. $\mathbf{p}^{P O S}$ and $\rho^{P O S}$ consist only one solution corresponds to the condition $p_{f}^{*} \geq p_{f}^{\max }$ and $p_{m}^{*} \geq p_{m}^{\max }$ or $\rho_{m}^{*} \geq \rho_{m}^{\max }$ and $\rho_{m}^{*}<\rho_{m}^{\max }$ and it implies that there is a unique universal optimum for case $(C 1)$. Hence, we only articulate on the condition corresponds to $p_{f}^{*}<p_{f}^{\max }$ and $p_{m}^{*}<p_{m}^{\max } \operatorname{or} \rho_{f}^{*}<\rho_{f}^{\max }$ and $\rho_{m}^{*}<\rho_{m}^{\max }$. Here, the multiempirical difficulty $(C 1)$ can be further extend as problem (C2) below:

$C 2: \max _{p_{f}, p_{m}, \rho_{f}, \rho_{m}} \phi_{S E}\left(p_{f}, p_{m}, \rho_{f}, \rho_{m}\right), \phi_{E E}\left(p_{f}, p_{m}, \rho_{f}, \rho_{m}\right)$, Subject to: $\left\{p_{f}, p_{m}\right\} \in \mathbf{p}^{P O S},\left\{\rho_{f}, \rho_{m}\right\} \in \rho^{P O S}$ where $\mathbf{p}^{P O S}=\left[p_{f}^{*}, p_{m}^{*} \leq\left\{p_{f}, p_{m}\right\} \leq\left\{p_{f}^{\max }, p_{m}^{\max }\right\}\right]$ and $\rho^{P O S}=\left[\rho_{f}^{*}, \rho_{m}^{*} \leq\left\{\rho_{f}, \rho_{m}\right\} \leq\left\{\rho_{f}^{\max }, \rho_{m}^{\max }\right\}\right]$.

For case $(C 2)$, the optimization scheme in [32]-[34] is invoke to find the distinctive universal optimum among the Pareto optimal nodes by fine-tuning the weighting metric $\theta$. $\phi_{S E}^{\max }$ and $\phi_{E E}^{\max }$ indicate optimum $\mathrm{SE}$ and $\mathrm{EE}$ in $p^{P O S}$ or $\rho^{P O S}$, respectively. To determine SE-EE trade-off, SE and $\mathrm{EE}$ converted into dimensionless metrics through the normalization method as follows:

$$
\phi_{S E}^{n o r m}=\frac{\phi_{S E}}{\phi_{S E}^{\max }},
$$

and

$$
\phi_{E E}^{n o r m}=\frac{\phi_{E E}}{\phi_{E E}^{\max }},
$$

where $\phi_{S E}^{\text {norm }}=\{0,1\}$ and $\phi_{E E}^{\text {norm }}=\{0,1\}$. Thus, by applying Cobb-Douglas production function [6], SE and EE can be presented in a utility function as below:

$$
U\left(\phi_{S E}, \phi_{E E}\right)=\left(\phi_{S E}^{n o r m}\right)^{\theta}\left(\phi_{E E}^{n o r m}\right)^{1-\theta} .
$$

It can be noticed from the above that the priority to maximize EE even more than SE for $\theta \rightarrow 0$ and the priority to maximize $\mathrm{SE}$ even more than $\mathrm{EE}$ for $\theta \rightarrow 1$. Hence, $(C 2)$ can be further extended to the single-empirical optimization case as presented below:

$$
\begin{aligned}
& C 3: \max _{p_{f}, p_{m}, \rho_{f}, \rho_{m}} U\left(\phi_{S E}, \phi_{E E}\right), \\
& \text { Subject to: } \left.\left\{p_{f}, p_{m}\right\} \in \mathbf{p}^{P O S},\left\{\rho_{f}, \rho_{m}\right\} \in \rho^{P O S}\right\} .
\end{aligned}
$$

For $C 3$, taking natural logarithmic transformation to (18), we get:

$$
\begin{aligned}
& \log _{e} U=\theta \log _{e} \phi_{S E}^{\text {norm }}+(1-\theta) \log _{e} \phi_{E E}^{\text {norm }} \\
& \text { or } Y=\theta \log _{e} \phi_{S E}+(1-\theta) \log _{e} \phi_{E E}-B,
\end{aligned}
$$

where $Y=\log _{e} U, B=\theta \log _{e} \phi_{S E}^{\max }+(1-\theta) \log _{e} \phi_{E E}^{\max }$ and it is a constant metric for the constant power and the fixed BS density.

Theorem 3: For fixed BS density, $C 3$ can further be presented for transmit power optimization in SE-EE trade-off as:

$$
C 4: \max _{p_{f}, p_{m}} Y\left(p_{f}, p_{m}\right) \text {, Subject to }:\left\{p_{f}, p_{m}\right\} \in \mathbf{p}^{P O S} \text {. }
$$

For $C 4, Y\left(p_{f}, p_{m}\right)$ is strictly quasi-concave with regard to $\mathbf{p}^{P O S}$.

Proof: Proof is delegated in Appendix D.

Theorem 4: For constant transmit power, $C 3$ can further be presented for BS density optimization in SE-EE trade-off as:

$$
C 5: \max _{\rho_{f}, \rho_{m}} Y\left(\rho_{f}, \rho_{m}\right) \text {, Subject to }:\left\{\rho_{f}, \rho_{m}\right\} \in \rho^{P O S} \text {. }
$$

For $C 5, Y\left(\rho_{f}, \rho_{m}\right)$ is strictly quasi-concave with regard to $\rho^{P O S}$.

(15) Proof: Proof is delegated in Appendix E. 


\section{Significance of $\theta$ in SE-EE trade-off:}

Based on (D.4) and (E.4), the effectiveness of SE-EE trade-off with regard to weighting factor, $\theta$, can be presented as follows:

(i) At $\theta=0$,

This condition is mainly to observe $\theta=0$ 's impact on EE. For fixed BS density, the optimal transmit power is $p_{f}^{o p t}=p_{f}^{* *}=p_{f}^{*}$ due to $\theta \leq 1-\phi\left(p_{f}^{\max }\right)$ or $p_{m}^{o p t}=p_{m}^{* *}=p_{m}^{*}$ due to $\theta \leq 1-\phi\left(p_{m}^{\max }\right)$; and for constant transmit power, the optimal BS density is $\rho_{f}^{o p t}=\rho_{f}^{* *}=\rho_{f}^{*}$ due to $\theta \leq 1-\phi\left(\rho_{f}^{\max }\right)$ or $\rho_{m}^{o p t}=\rho_{m}^{* *}=\rho_{m}^{*}$ due to $\theta \leq 1-\phi\left(\phi_{m}^{\max }\right)$

\section{(ii) At $\theta=1$,}

This condition is mainly to observe $\theta=1$ 's impact on SE. For fixed BS density, the optimal transmit power is $p_{f}^{o p t}=p_{f}^{\max }$ due to $\theta>1-\phi\left(p_{f}^{\max }\right.$ or $p_{m}^{o p t}=p_{m}^{\max }$ due to $\theta>1-\phi\left(p_{m}^{\max }\right)$; for constant transmit power, the optimal BS density is $\rho_{f}^{o p t}=\rho_{f}^{\max }$ due to $\theta>1-\phi\left(\rho_{f}^{\max }\right)$ or $\rho_{m}^{o p t}=\rho_{m}^{\max }$ due to $\theta>1-\phi\left(\rho_{m}^{\max }\right)$.

(iii) At $0<\theta<1$,

This condition is to observe $0<\theta<1$ 's impacts on both SE and EE. By fine-tuning the weighting factor $\theta$, the optimum result can be achieved by exploiting $U\left(p_{f}, p_{m}, \rho_{f}, \rho_{m}\right)$, i.e., by maximizing transmit power with fixed BS density in Theorem 3 and by maximizing BS density with constant transmit power in Theorem 4.

\section{NumERICAL RESUltS AND Discussion}

This unit illustrates the analytical results along with its Monte Carlo simulation results, where the simulation results vindicate the analytical results computed applying the developed mathematical expressions in unit II. The considered network metrics for the dual-layer networks, consisting of randomly distributed femtocells and macrocells, are taken as follows: $B W=25 \mathrm{MHz}, b=450 \mathrm{kHz}, \alpha=4, \rho_{f}=8 \times 10^{-4}$ $\mathrm{FBSs} / \mathrm{m}^{2}, \rho_{m}=4 \times 10^{-2} \mathrm{MBSs} / \mathrm{km}^{2}, \mathrm{p}_{f}=45 \mathrm{dBm}, p_{m}=$ $75 \mathrm{~dB}, \phi_{f}=0.8 \mathrm{Mbps}, \phi_{m}=0.8 \mathrm{Mbps}, a_{f}=8.0, a_{m}=3.4$, $b_{f}=5.2 \mathrm{~W}, b_{m}=65 \mathrm{~W}, r=20 \mathrm{~m}$.

Figs 2 and 3 represent the response of the SE and EE as a function of the ratio of $\rho_{m}$ to $S_{g}$ under the different ratios of $\rho_{f}$ to $\rho_{m}$, respectively. It is illustrated in Fig. 2 that while $S_{g}$ is provided, the curve representing SE grows with $\frac{\rho_{m}}{S}$. For a known value of $\frac{\rho_{m}}{S_{g}}$, the SE become heightens with $\frac{\rho_{f}}{\rho_{m}}$ in Fig. 2. This is due to the fact that, the throughput of the cellular HetNets gets bigger with the grow in $\frac{\rho_{f}}{\rho_{m}}$. Nevertheless, while the numerical value of $\frac{\rho_{f}}{\rho_{m}}$ exceeds a certain threshold level, the SE grows at a slow pace than earlier. This is due to the fact that, escalating the value of $\frac{\rho_{f}}{\rho_{m}}$ produces even more unwanted interfering BSs near to the MU. Fig. 3 depicts that, for different $\frac{\rho_{f}}{\rho_{m}}$, the EE gets bigger with $\frac{\rho_{m}}{S_{g}}$ to a peak point and then again begins to get smaller. As with the increase in MBS, simultaneously the overall power utilization in the network

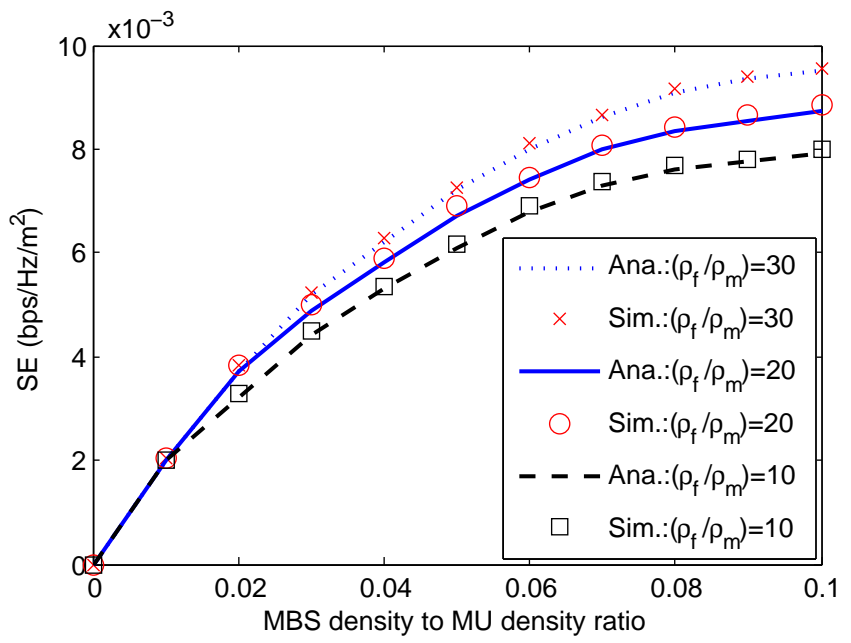

Fig. 2: Dual layers SE vs. MBS to MU density ratio for different ratios of FBS to MBS density

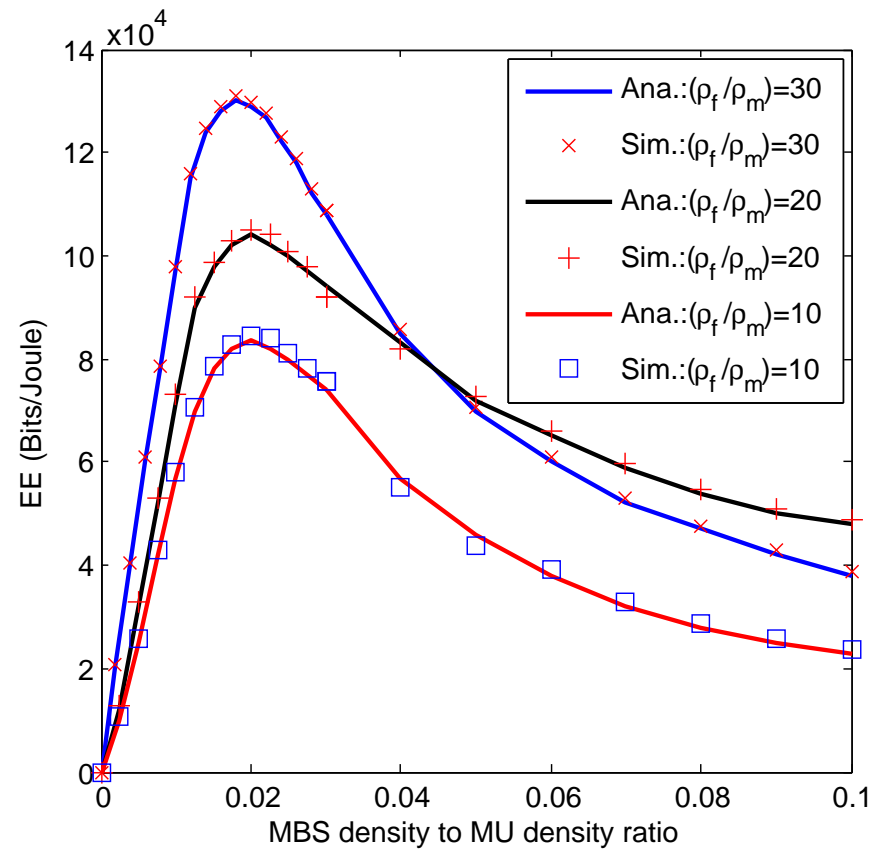

Fig. 3: Dual layers EE vs. MBS to MU density ratio for different ratios of FBS to MBS density

gets higher. Besides, it can be noticed that the escalation of throughput is slow-going compare to the escalation of energy utilization. Also, the EE grows with $\frac{\rho_{f}}{\rho_{m}}$ while $\frac{\rho_{m}}{S_{g}}$ is provided. A cause behind this is that FBSs privileged MUs increase with $\frac{\rho_{f}}{\rho_{m}}$ for a certain numerical value of $\frac{\rho_{m}}{S_{g}}$, which helps to achieve more network throughput. Figs. 2 and 3 also demonstrate that, for different ratio of $\frac{\rho_{f}}{\rho_{m}}$, an optimal $\rho_{m}$ and a respective $\rho_{f}$ can be achieved to obtain optimum EE maintaining the minimal desired SE.

An impression of SE and EE as a function of the ratio of $\rho_{f}$ to $\rho_{m}$ under the different ratios of $\rho_{m}$ to $S_{g}$ is presented in Figs. 4 and 5, respectively. The graphical response curves of the SE are depited in Figure 4 and the cause remain same to that of Fig. 2. In Fig. 5, one can remark that the EE initially grows with $\frac{\rho_{f}}{\rho_{m}}$ and then reduces for know value of $\frac{\rho_{m}}{S_{g}}$. While 


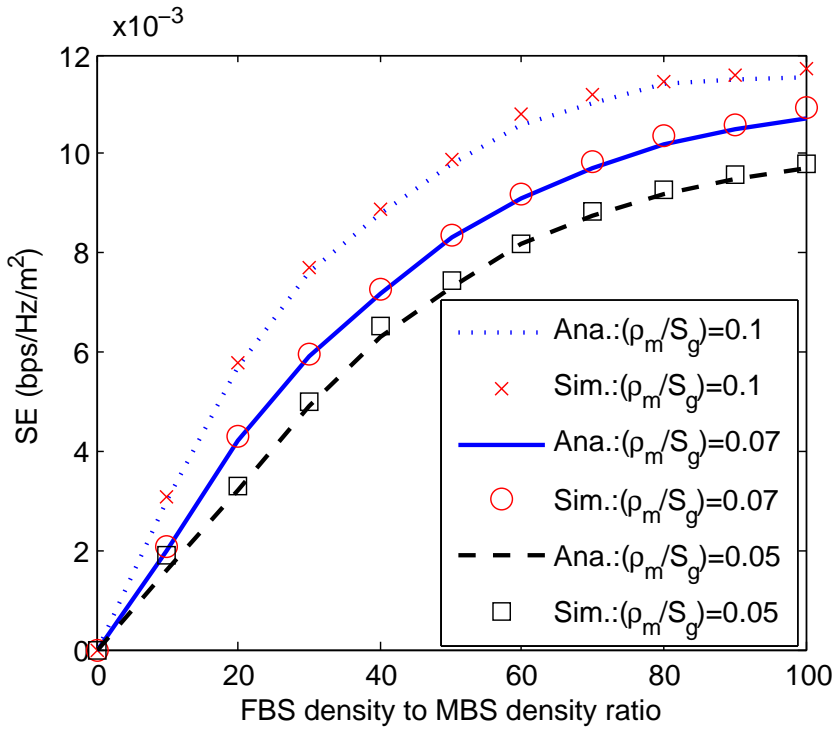

Fig. 4: Dual layers SE vs. FBS to MBS density ratio for different ratios of MBS to MU density

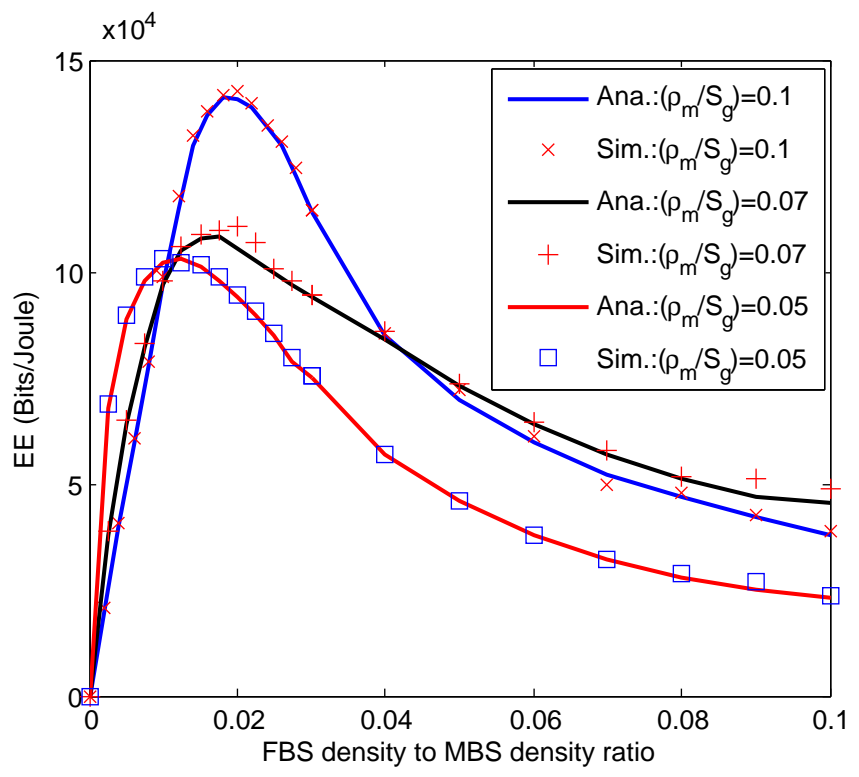

Fig. 5: Dual layers EE vs. FBS to MBS density ratio for different ratios of MBS to MU density

$\frac{\rho_{f}}{\rho_{m}}$ is smaller than a certain level, then for a given $\frac{\rho_{m}}{S_{g}}$, a larger $\rho_{m}$ results in a higher EE for a fixed value of $\frac{\rho_{f}}{\rho_{m}}$. Likewise, while $\frac{\rho_{f}}{\rho_{m}}$ is higher than a certain level, a larger $\rho_{m}$ results in a lesser EE for a fixed value of $\frac{\rho_{f}}{\rho_{m}}$. The cause of these results is that while $\frac{\rho_{f}}{\rho_{m}}$ increases, the total BSs is seemed to grow which results in large power utilization and unwanted interfering BSs. Furthermore, the energy utilization of a MBS is even very large in contrast to a FBS. Additionally, this can be drawn to a conclusion that if $S_{g}$ and $\frac{\rho_{m}}{S_{g}}$ are known, then it is possible to determine the optimal $\frac{\rho_{f}}{\rho_{m}}$ to achieve optimum EE maintaining the minimal desired $\stackrel{\rho_{m}}{\mathrm{SE}}$.

In the SE-EE trade-off case studies while the transmit power is optimized keeping $\rho_{f}=\rho_{f}^{\max }$ fix, and assuming $\rho_{f}^{\max }=135$ and $p_{f}^{\max }=2.3 \mathrm{~W}$, fig. 6 illustrates the role of weighting factor, $\theta$, to reach the optimal transmit power.
The three graphical plots with respect to the different SINR threshold, $\varphi$, all exhibit that $p_{f}^{o p t}$ initially grows with $\theta$ and then remains unaltered with $\theta$ gets bigger up to a certain level, which satisfies well with $(D .3)$.

In the SE-EE trade-off case studies while the BS density is optimized with constant transmit power, and assuming $\rho_{f}^{\max }=$ 135 and $p_{f}^{\max }=1.8 \mathrm{~W}$, fig. 7 illustrates the role of weighting factor, $\theta$, to reach the optimal BS density. The three graphical plots with respect to a fixed SINR threshold, $\varphi$, all exhibit that $\theta_{f}^{o p t}$ initially grows with $\theta$ and then remains unaltered with $\theta$ gets bigger up to a certain level, ranging from $\rho_{f}^{*}$ to $\rho_{f}^{\max }$, which satisfies well with (E.3).

Fig. 8 aims to illustrate the significance of SINR threshold 2 on the SE-EE trade-off to establish the interrelationship between the network SE and the network EE. It can be revealed that initially SE and EE grow concurrently, indicating no compromise between two desirable but incompatible features in an intervening time as no node in this duration is superior than the corresponding counterpart with the exactly similar EE positioned in the rest part of duration. The EE-SE tradeoff cannot reached until and unless the optimal node appears, after that EE reduces with SE. Particularly, from Figs. 6, 7 and 8 , it can be seen that maximum EE $\phi_{E E}^{\max }=14.25 \times 10^{4} \mathrm{bits} / \mathrm{J}$ is achieved at $\theta=0$ when $p_{f}^{o p t}=250 \mathrm{~mW}$ and $\rho_{f}^{o p t}=135$, whereas the maximum SE $\phi_{S E}^{\max }=6 \times 10^{-3} \mathrm{bps} / \mathrm{Hz} / \mathrm{m}^{2}$ is achieved at $\theta=0$ when $p_{f}^{o p t}=p_{f}^{\max }=250 \mathrm{~mW}$ and $\rho_{f}^{o p t}=\rho_{f}^{\max }=135$, which harmonizes satisfactorily with the developed analytical framework in section III. However, it can be seen that the EE-SE trade-off can be reached by finetuning $\theta$ from 0 to 1 , i.e., $\phi_{E E}^{\max }$ is shifting from $14.25 \times 10^{4}$ bits/J to $5.3 \times 10^{4}$ bits/J , whilst $\phi_{S E}^{\max }$ is shifting from $4.98 \times 10^{-3} \mathrm{bps} / \mathrm{Hz} / \mathrm{m}^{2}$ to $10 \times 10^{-3} \mathrm{bps} / \mathrm{Hz} / \mathrm{m}^{2}$. These results can directly give some insight to regulate the power and BS density in dual-layers network to obtain the precise $\phi_{E E}$ and $\phi_{S E}$, for instance if an estimated $\phi_{E E}$ of $9.6 \times 10^{4}$ bits/ $\mathbf{J}$ is needed, then we can adjust $\theta$ to 0.5 by incorporating the respective optimal transmit power $p_{f}^{o p t}=1.6 \mathrm{~W}$ and the respective optimal BS density $\rho_{f}^{o p t}=135$. It is undoubtedly aid to minimize the energy utilization. The node where the network shortcomings account into the tradeoff establishment is signalized by an explicit $\mathrm{SE}$ value after that the EE response deteriorates. Although, for $I=15 d B$, reduction in the EE is not that much observable as the highest obtainable EE is

${ }^{2}$ It is a metric to take on radio condition of Wireless links in the heterogeneous cellular networks. Due to noise and interference intensity of different concurrent transmission, path-loss takes into account and it increases with the increase in distance which turns in the energy of the signal gets faded with distance. In general, radio condition can be measured based on the following numerical values of SINR as listed below: (0 to 6) $\mathrm{dB}$ is treated as a poor radio condition, ( 7 to 16 ) $\mathrm{dB}$ is treated as an average radio condition, (17 to 26$) \mathrm{dB}$ is treated as a good radio condition, ( $27 \mathrm{~dB}$ and above) is treated as an excellent radio condition.

In addition, Reference signal receive power, i.e., numerator part of SINR, is one of the fundamental metrics of the user equipment physical layer characteristics to greatest extent and this is also a linear average power of a reference signal in DL transmission on the channel for the cognitive radio resource blocks which bears macrocell/femtocell specific reference signals. The information of the concerned parameter gives the idea on the degree of intensity of macrocell/femtocell and it helps to compute path loss to apply in the operation for deciding the most appropriate power settings to functioning the network. 


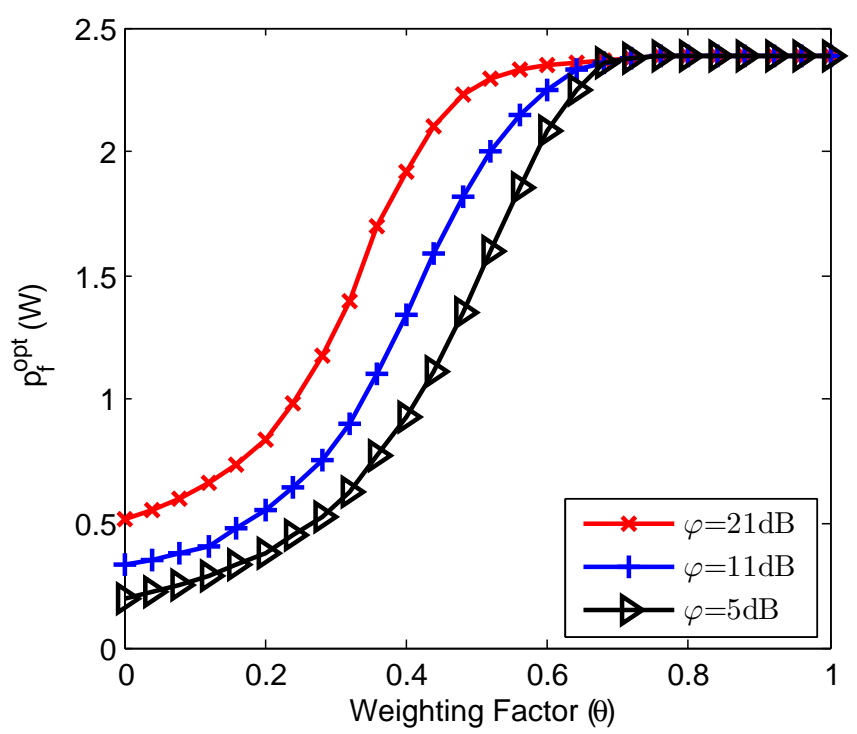

Fig. 6: Optimal transmit power vs. weighting factor for different SINR thresholds

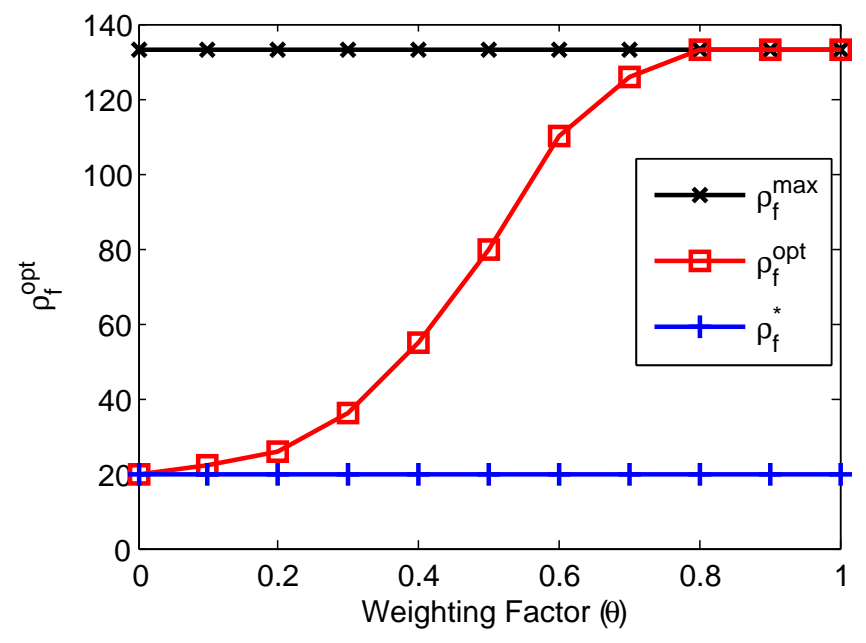

Fig. 7: Optimal FBS density vs. weighting factor for fixed SINR threshold

restricted by the interference. Nevertheless, co-tier and crosstier interference produce remarkable impact on the SE-EE trade-off response, which is making it different from that illustrated in [6]. More precisely, the SE-EE trade-off analysis in [6] had not considered the dual-layers network, which describes the dissimilitude.

Fig. 9 illustrates the EE and SE tradeoff curves of transmission links for $p_{f}^{o p t}=400,250 \mathrm{~mW}$, respectively. For individual link, the SE demand is grown from 0 to $12 \times 10^{-3} \mathrm{bps} / \mathrm{Hz} / \mathrm{m}^{2}$ with a step of 2, and the respective EE is achieved by Theorem 2. For any defined SE demand $\left(0 \leq U\left(\phi_{S E}, \phi_{E E}\right) \leq 12 \times\right.$ $\left.10^{-3} \mathrm{bps} / \mathrm{Hz} / \mathrm{m}^{2}\right)$, a network has always opportunity to fulfill the SE demand if the transmission link gain of the wanted signal is big enough in contrast to the unwanted interference signal gain. Simulation results demonstrate that the highest obtainable EE is restricted by $p_{f}^{o p t}$, which is mainly valid in the upper SE region. If the fixed power-offset is incorporated into the account, as given in (7) and (8), the EE grows initially and then reduces as SE move towards the upper region.

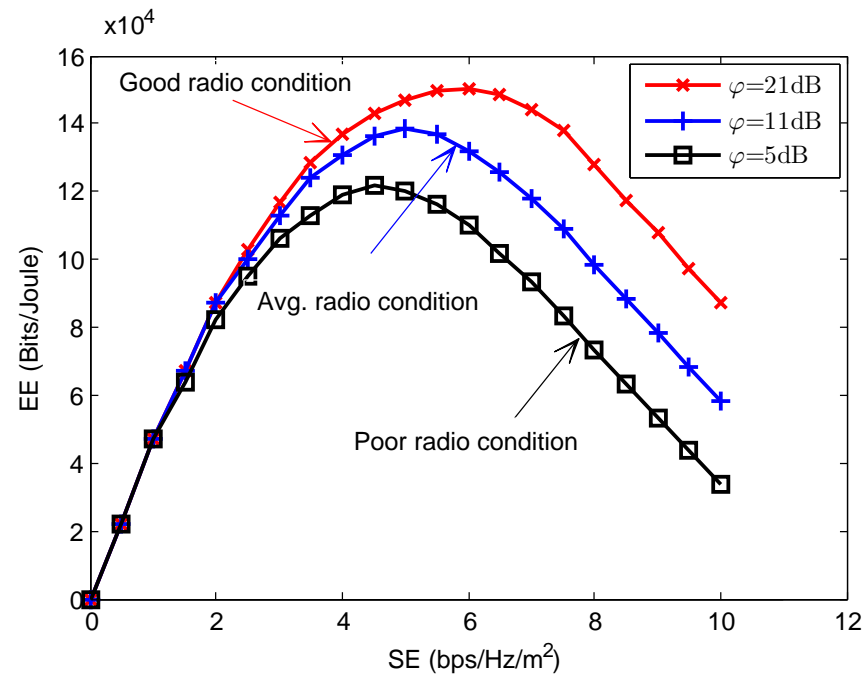

Fig. 8: Dual layer EE as a function of dual layers SE for different SINR thresholds

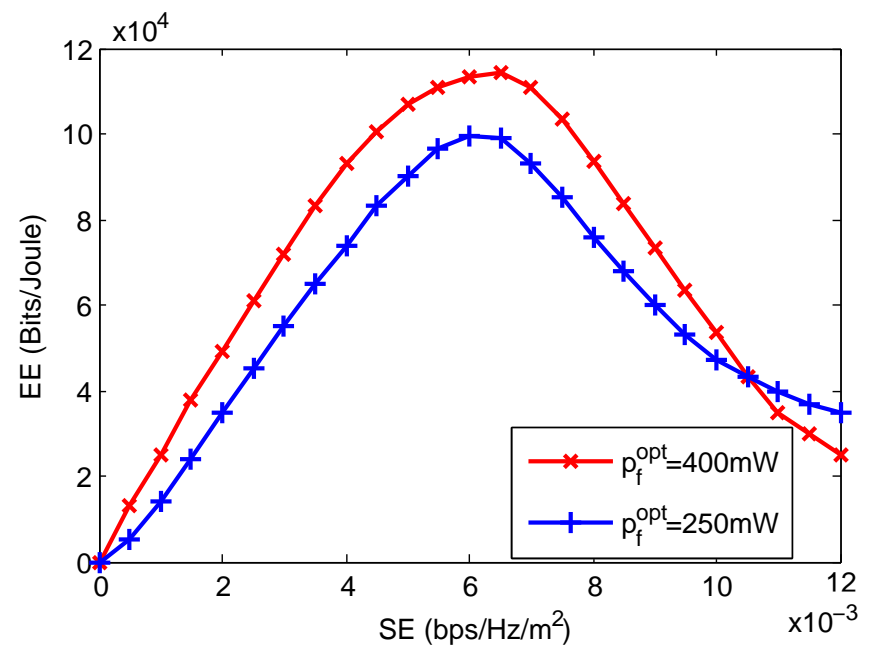

Fig. 9: Dual layer EE as a function of dual layers SE for different optimal transmit powers

\section{CONCLUSiON}

This paper provides the framework of two independent spatial PPP distribution to investigate the trade-off between SE and EE metrics of OFDMA based macro-femtocell networks in DL transmission, which is eventually expressed in a concise way by POS based on multi-empirical optimization issue. A utility function has been developed applying the Cobb-Douglas production function by which multi-empirical optimization issue is transformed onto a single-empirical optimization case via weighting factor. By analytical and simulation results, it is shown the significant role of optimal transmit power and SINR threshold in the response of EE as a function of SE.

\section{APPENDIX A}

Proof of EQUATION No.(2)

The derivation of SP in DL communication is obtained by following the step outlined in [26]. Applying (1) in the 
definition of SP, we get:

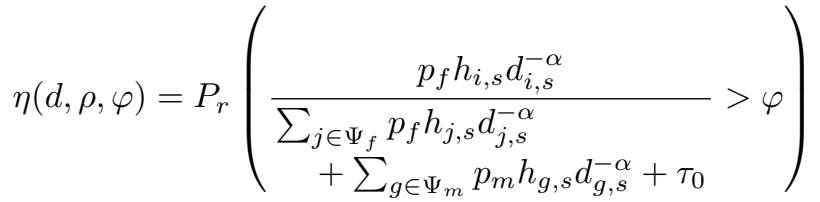

$$
\begin{aligned}
& =P_{r}\left(h_{i, s}>\frac{\varphi d_{i, s}^{\alpha}}{p_{f}}\left(I_{f}+I_{m}+\tau_{0}\right)\right) \\
& =E\left[\exp \left(\frac{\varphi d_{i, s}^{\alpha}}{p_{f}}\left(I_{f}+I_{m}+\tau_{0}\right)\right)\right],
\end{aligned}
$$

where $P_{r}(m)$ stands for the probability of the event $m$ and $E[q]$ stands for the expectation of the random variable $q, h_{i, s}$ represents SSF coefficient (considered to be Rayleigh faded) of the channel between $i^{t h}$ FBS and $S_{i}^{t h}$ FU and its power is exponentially distributed with mean $\frac{1}{\mu}, \forall i$, where $\mu$ indicates Rayleigh distribution. Followed by [29], Laplace transform (LT) of inter-femtocell interference, $I_{f}=\sum_{j \in \Psi_{f}} p_{f} h_{j, s} d_{j, s}^{-\alpha}$, on the RBG allotted to $S_{i}^{t h}$ FU by $i^{t h}$ FBS can be expressed as:

$$
\begin{aligned}
L T\left\{I_{f}\right\} & =E_{I}\left[\exp \left(-s I_{f}\right)\right]=E_{\Psi_{f}}\left[\Pi_{j \in \Psi_{f}} \exp \left(-s p_{f} h_{j, s} d_{j, s}^{-\alpha}\right)\right] \\
& =\exp \left[-2 \pi \rho_{f} \int_{0}^{\infty} 1-L_{h}\left(s p_{f} c^{-\alpha}\right)\right] \\
& =\exp \left[-2 \pi \rho_{f} \int_{0}^{\infty} \frac{c}{1+\mu c^{\alpha} s^{-1}} d c\right]
\end{aligned}
$$

where $(A .2)$ is obtained by considering the PPPs probability generating function (PGF) [25], and $(A .3)$ follows the exponential distribution of $h_{j, s}$. By replacing, $s=\frac{\varepsilon \varphi d^{\alpha}}{P_{f}}$, the LT of $I_{f}$ can be expressed as:

$$
L_{I_{f}}\left(\varepsilon \frac{\varphi d^{\alpha}}{P_{f}}\right)=\exp \left[-2 \pi \rho_{f} \int_{0}^{\infty} \frac{c}{1+c^{\alpha} d^{-\alpha} \varphi^{-1}} d c\right] .
$$

Applying $(A .4)$ in $(A .1)$, we get:

$$
\eta(d, \rho, \varphi)=L_{I_{f}}\left(\varepsilon \frac{\varphi d^{\alpha}}{P_{f}}\right) \exp \left(\frac{\zeta}{p_{f}}\right),
$$

where $\zeta=\varepsilon \varphi d^{\alpha} \tau_{0}$. The Simplification of $(A .5)$ gives [26]:

$$
\eta(d, \rho, \varphi)=\exp \left[-\rho_{f} \xi_{\alpha}-\frac{\zeta}{p_{f}}\right]
$$

where $\xi_{\alpha}=d^{2} \varphi^{\frac{2}{\alpha}} \lambda_{\alpha}, \lambda_{\alpha}=2 \pi \Gamma\left(\frac{2}{\alpha}\right) \Gamma\left(1-\frac{2}{\alpha}\right) \alpha^{-1}$ and $\Gamma(m)$ being the Gamma function of $m$.

\section{APPENDIX B}

\section{PROOF OF THEOREM 1}

To postulate the possessions of (3), we follow the statement on quasi-concavity in [30]. Function $f: \mathbf{A} \rightarrow \mathbf{D}$ defined on the interval $\mathbf{A} \subset \mathbf{D}$ strictly quasi-concave if and only if, $a_{1} \neq a_{2} \forall a_{1}, a_{2}$, i.e.,

$$
f\left(u a_{1}-(1-u) a_{2}\right)>\min \left\{f\left(a_{1}\right), f\left(a_{2}\right)\right\},
$$

where $u$ is a weight metrics variation on a particular scale of $(0,1)$. Likewise, for any certain value $y$ as $f\left(a_{1}\right)>y$ and $f\left(a_{2}\right)>y$, thus this can be deduced as $f\left(u a_{1}-(1-u) a_{2}\right)>y$ [31]. From the denotation of SE as defined in (3), the corresponding function which can be applied in the analysis as $f(a)=m_{1} \exp \left(-v_{1} a\right)+m_{2} a \exp \left(-v_{2} a\right),\left\{v_{1}, v_{2}, m_{1}, m_{2}\right\} \in$ $\mathbf{D}$, and $a \geq 0$.

Obeying the method given in [31], this can be concluded that (3) never content outside the scale of $y=(0, Q), Q$ is the highest value of $f(a)$ obtained at the maximum point $a^{*}$, hence no points lying for the conditions $a<0$ and $a>Q$. For the points lying within the scale $(0, Q)$, this can be followed that, provided the continuity of $f(a)$, there lies specific corresponding values $a^{\prime}$ and $a^{\prime \prime}$, so that $f(a)>y, \forall a$ in the interim $a^{\prime}<a<a^{\prime \prime}$. Hence, for provided $a_{1}$ and $a_{2}$ distinguished by $0 \leq a_{1}<a_{2}$ for that $f\left(a_{1}\right)>y$ and $f\left(a_{2}\right)>y$, it obeys the conditions $a^{\prime}<u a_{1}-(1-u) a_{2}<a^{\prime \prime}$ and $f\left(u a_{1}-(1-u) a_{2}\right)>y$ for $0<u<1$. As $f(a)$ is strictly quasi-concave in the region $[0, \infty]$, the regional optimal point at $a^{*}$ is also the universal optimum.

\section{APPENDIX C \\ PROOF OF THEOREM 2}

From the denotation of EE as defined in (7), the corresponding function which can be applied in the analysis as: $f(a)=\frac{m_{1} \exp \left(-v_{1} a\right)+m_{2} a \exp \left(-v_{2} a\right)}{a+c},\left\{v_{1}, v_{2}, m_{1}, m_{2}, c\right\} \in \mathbf{D}$ and $a \geq 0$. When the framework of the numerator $m_{1}$ $\exp \left(-v_{1} a\right)+m_{2} a \exp \left(-v_{2} a\right)$ is giving a detailed account in Appendix $B$, the denominator $(a+c)$ is a linear function and also strictly quasi-concave. According to the definition of strict quasi-concavity, for any $0 \leq a_{1}, a_{2}<1$ and $a_{1} \neq a_{2}$, it can be followed as:

$$
\begin{aligned}
& \begin{array}{l}
m_{1} \exp \left(-v_{1}\left(u a_{1}-(1-u) a_{2}\right)\right) \\
\quad+m_{2}\left(u a_{1}-(1-u) a_{2}\right) \exp \left(-v_{2}\left(u a_{1}-(1-u) a_{2}\right)\right) \\
\left(u a_{1}-(1-u) a_{2}\right)+c
\end{array} \\
& >\min \left[\frac{m_{1} \exp \left(-v_{1} a_{1}\right)+m_{2} a_{1} \exp \left(-v_{2} a_{1}\right)}{a_{1}+c}\right. \\
& \left.\frac{m_{1} \exp \left(-v_{1} a_{2}\right)+m_{2} a_{2} \exp \left(-v_{2} a_{2}\right)}{a_{2}+c}\right]
\end{aligned}
$$

where $c>0$. Thus, to provide the existence of the universally optimum for the strict quasi-concave function, the maximal metric $a^{*}$ to be achieved by considering the derivative of $f(a)$ and adjusting this equals to zero, and then computing for $a^{*}$.

\section{APPENDIX D}

By taking the 1st derivative of $Y\left(p_{f}\right)$ with respect to $p_{f}$, we get:

$$
Y^{\prime}\left(p_{f}\right)=\theta \frac{\phi_{S E}^{\prime}}{\phi_{S E}}+(1-\theta) \frac{\phi_{E E}^{\prime}}{\phi_{E E}} .
$$

Let $Y^{\prime}\left(p_{f}\right)=0$, which provides:

$$
\theta=\frac{\phi_{E E}^{\prime} \phi_{S E}}{\phi_{E E}^{\prime} \phi_{S E}-\phi_{S E}^{\prime} \phi_{E E}}
$$




$$
\text { or, }(1-\theta)=\frac{\phi_{S E}^{\prime} \phi_{E E}}{\phi_{E E}^{\prime} \phi_{S E}-\phi_{S E}^{\prime} \phi_{E E}} \text {. }
$$

As $\theta \in[0,1]$, two case studies can be presented below:

(i) $\theta>\left(1-\phi\left(p_{f}^{\max }\right)\right)$

In this case $(1-\theta)<\phi\left(p_{f}^{\max }\right) \leq \phi\left(p_{f}\right)$, then $\phi\left(p_{f}\right)=$ $(1-\theta)$ has no solution, and signifying $Y^{\prime}\left(p_{f}\right)>0$.

Hence $Y\left(p_{f}\right)$ is an increasing function of $p_{f}$ in $\mathbf{p}^{P O S}$.

(ii) $\theta \leq\left(1-\phi\left(p_{f}^{\max }\right)\right)$

In this case $\phi\left(p_{f}^{\max }\right) \leq(1-\theta) \leq 1$. As $\phi\left(p_{f}\right)$ decreases with the increase in $p_{f}$ and $\phi\left(p_{f}^{*}\right)=1,(D .2)$ has one and only one solution and it is indicated by $p_{f}^{* *}$ for convenience. Therefore POS can be split up into parts by $p_{f}^{* *}$. In one part $\left[p_{f}^{*}, p_{f}^{* *}\right], Y^{\prime}\left(p_{f}\right)>0$ holds with $\phi\left(p_{f}\right)-(1-\theta)>0$. While in the second part $\left[p_{f}^{* a s t}, p_{f}^{\max }\right], Y^{\prime}\left(p_{f}\right)<0$ holds with $\phi\left(p_{f}\right)-$ $(1-\theta)<0$. Thus $Y\left(p_{f}\right)$ initially grows and then reduces with the increase of $p_{f}$ in $\mathbf{p}^{P O S}$.

Hence $Y\left(p_{f}\right)$ is strictly quasi-concave in $\mathbf{p}^{P O S}$. The optimal solution for $C 4$ can be presented as below:

$$
p_{f}^{o p t}= \begin{cases}p_{f}^{* *} ; & \theta \leq\left(1-\phi\left(p_{f}^{\max }\right)\right) \\ p_{f}^{\max } ; & \theta>\left(1-\phi\left(p_{f}^{\max }\right)\right) .\end{cases}
$$

Likewise, $Y\left(p_{m}\right)$ is strictly quasi-concave in $\mathbf{p}^{P O S}$. The optimal solution for $C 4$ can be presented as below:

$$
p_{m}^{o p t}= \begin{cases}p_{m}^{* *} ; & \theta \leq\left(1-\phi\left(p_{m}^{\max }\right)\right) \\ p_{m}^{\max } ; & \theta>\left(1-\phi\left(p_{m}^{\max }\right)\right) .\end{cases}
$$

\section{ApPendix E}

By taking the 1st derivative of $Y\left(\rho_{f}\right)$ with respect to $\rho_{f}$, we get:

$$
Y^{\prime}\left(\rho_{f}\right)=\theta \frac{\phi_{S E}^{\prime}}{\phi_{S E}}+(1-\theta) \frac{\phi_{E E}^{\prime}}{\phi_{E E}} .
$$

Let $Y^{\prime}\left(\rho_{f}\right)=0$, which provides:

$$
\begin{gathered}
\theta=\frac{\phi_{E E}^{\prime} \phi_{S E}}{\phi_{E E}^{\prime} \phi_{S E}-\phi_{S E}^{\prime} \phi_{E E}}, \\
\text { or, }(1-\theta)=\frac{\phi_{S E}^{\prime} \phi_{E E}}{\phi_{E E}^{\prime} \phi_{S E}-\phi_{S E}^{\prime} \phi_{E E}} .
\end{gathered}
$$

As $\theta \in[0,1]$, two case studies can be presented below:

(i) $\theta>\left(1-\phi\left(\rho_{f}^{\max }\right)\right)$

In this case $(1-\theta)<\phi\left(\rho_{f}^{\max }\right) \leq \phi\left(\rho_{f}\right)$, then $\phi\left(\rho_{f}\right)=$
Hence $Y\left(\rho_{f}\right)$ is an increasing function of $\rho_{f}$ in $\rho^{P O S}$.

(ii) $\theta \leq\left(1-\phi\left(\rho_{f}^{\max }\right)\right)$

In this case $\phi\left(\rho_{f}^{\max }\right) \leq(1-\theta) \leq 1$. As $\phi\left(\rho_{f}\right)$ decreases with the increase in $\rho_{f}$ and $\phi\left(\rho_{f}^{*}\right)=1,(E .2)$ has one and only one solution and it is indicated by $\rho_{f}^{* *}$ for convenience. Therefore POS can be split up into parts by $\rho_{f}^{* *}$. In one part $\left[\rho_{f}^{*}, \rho_{f}^{* *}\right], Y^{\prime}\left(\rho_{f}\right)>0$ holds with $\phi\left(\rho_{f}\right)-(1-\theta)>0$. While in the second part $\left[\rho_{f}^{* a s t}, \rho_{f}^{\max }\right], Y^{\prime}\left(\rho_{f}\right)<0$ holds with $\phi\left(\rho_{f}\right)-$ $(1-\theta)<0$. Thus $Y\left(\rho_{f}\right)$ initially grows and then reduces with the increase of $\rho_{f}$ in $\rho^{P O S}$.

Hence $Y\left(\rho_{f}\right)$ is strictly quasi-concave in $\mathbf{p}^{P O S}$. The optimal solution for $C 4$ can be presented as below:

$$
\rho_{f}^{o p t}= \begin{cases}{\left[\rho_{f}^{* *}\right] ;} & \theta \leq\left(1-\phi\left(\rho_{f}^{\max }\right)\right) \\ \rho_{f}^{\max } ; & \theta>\left(1-\phi\left(\rho_{f}^{\max }\right)\right) .\end{cases}
$$

where the symbol [.] indicates that $\rho_{m}^{o p t}$ is either nearest smaller integer or nearest greater integer to $\rho_{f}^{* *}$.

Likewise, $Y\left(\rho_{m}\right)$ is strictly quasi-concave in $\rho^{P O S}$. The optimal solution for $C 5$ can be presented as below:

$$
\rho_{m}^{o p t}= \begin{cases}{\left[\rho_{m}^{* *}\right] ;} & \theta \leq\left(1-\phi\left(\rho_{m}^{\max }\right)\right) \\ \rho_{m}^{\max } ; & \theta>\left(1-\phi\left(\rho_{m}^{\max }\right)\right) .\end{cases}
$$

\section{REFERENCES}

[1] Yun Li, Haluk Celebi, Mahmoud Daneshmand, Chonggang Wang, Weiliang Zhao, "Energy-efficient femtocell networks: challenges and opportunities," IEEE Wireless Communications, vol. 20, no. 6, pp. 99$105,2013$.

[2] Guogang Zhao, Sheng Chen, Liqiang Zhao, Lajos Hanzo, "Joint EnergySpectral-Efficiency Optimization of CoMP and BS Deployment in Dense Large-Scale Cellular Networks," IEEE Transactions on Wireless Communications, vol. 16, no. 7, pp. 4832-4847, 2017.

[3] Chan-Ching Hsu, J. Morris Chang, "Spectrum-Energy Efficiency Optimization for Downlink LTE-A for Heterogeneous Networks," IEEE Transactions on Mobile Computing, vol. 16, no. 5, pp. 1449-1461, 2017

[4] Qi Ren, Jiancun Fan, Xinmin Luo, Zhikun Xu, Yami Chen, "Energy Efficient Base Station Deployment Scheme in Heterogeneous Cellular Network," IEEE 81st Vehicular Technology Conference (VTC Spring) 2015, pp. 1-5, 2015.

[5] J. Ghosh, D. N. K. Jayakody, "An Analytical View of ASE for Multicell OFDMA Networks Based on Frequency Reuse Scheme," IEEE Systems Journal, July 2018

[6] Jaya B. Rao, Abraham O. Fapojuwo, "On the Tradeoff between Spectral Efficiency and Energy Efficiency of Homogeneous Cellular Networks with Outage Constraint," IEEE Transactions on Vehicular Technology, Vol. 62, No. 4, 2013.

[7] N. Saquib, E. Hossain, D. Kim, "Fractional frequency reuse for interference management in LTE-advanced hetnets," IEEE Wireless Commun. pp. 113-122, 2013.

[8] Hesham ElSawy, Ahmed Sultan-Salem, Mohamed-Slim Alouini, Moe Z. Win, "Modeling and Analysis of Cellular Networks Using Stochastic Geometry: A Tutorial," IEEE Communications Surveys and Tutorials, vol. 19, no. 1, pp. 167-203, 2017.

[9] Ahmad AlAmmouri, Jeffrey G. Andrews, Franois Baccelli, "SINR and Throughput of Dense Cellular Networks With Stretched Exponential Path Loss," IEEE Transactions on Wireless Communications, vol. 17, no. 2, pp. 1147-1160, 2018.

[10] Murtadha Al-Saedy, Hamed Al-Raweshidy, Hussien Al-Hmood, Fourat Haider, "Coverage and Effective Capacity in Downlink MIMO Multicell Networks With Power Control: Stochastic Geometry Modelling," IEEE Access, vol. 6, pp. 9173-9185, 2018. 
[11] Chenlong Jia, Teng Joon Lim, "Designing femtocell exclusion zones to minimize power in a heterogeneous network," Global Communications Conference (GLOBECOM) 2014 IEEE, pp. 4251-4256, 2014.

[12] Mehdi Fereydooni, Masoud Sabaei, Mehdi Dehghan, Martin Taranetz, Markus Rupp, "A Mathematical Framework to Evaluate Flexible Outdoor User Association in Urban Two-Tier Cellular Networks," IEEE Transactions on Wireless Communications, vol. 17, no. 3, pp. 15591573, 2018.

[13] Younggap Kwon, Taewon Hwang, Xudong Wang, "Energy-Efficient Transmit Power Control for Multi-tier MIMO HetNets," IEEE Journal on Selected Areas in Communications, vol. 33, no. 10, pp. 2070-2086, 2015.

[14] Shunqing Zhang, Qingqing Wu, Shugong Xu, Geoffrey Ye Li,"Fundamental Green Tradeoffs: Progresses Challenges and Impacts on 5G Networks," Communications Surveys Tutorials IEEE, vol. 19, no. 1 , pp. 33-56, 2017

[15] Kyuho Son, Eunsung Oh, Bhaskar Krishnamachari,"Energy-efficient design of heterogeneous cellular networks from deployment to operation," Computer Networks, vol. 78, pp. 95, 2015.

[16] S. Buzzi, G. Colavolpe, D. Saturnino, A. Zappone, "Potential games for energy-efficient power control and subcarrier allocation in uplink multicell OFDMA systems," IEEE J. Topics Signal Process., vol. 6, no. 2, pp. 89-103, Apr. 2012.

[17] Muhammad Ismail, Amila Tharaperiya Gamage, Weihua Zhuang, Xuemin Sherman Shen, "Energy efficient uplink resource allocation in a heterogeneous wireless medium," Communications (ICC) 2014 IEEE International Conference on, pp. 5275-5280, 2014.

[18] Fei Liu, Kan Zheng, Wei Xiang, Hui Zhao, "Design and Performance Analysis of An Energy-Efficient Uplink Carrier Aggregation Scheme,' IEEE Journal on Selected Areas in Communications, vol. 32, no. 2, pp 197-207, 2014

[19] Chun-Hung Liu, Kok Leong Fong, "Fundamentals of the Downlink Green Coverage and Energy Efficiency in Heterogeneous Networks,' IEEE Journal on Selected Areas in Communications, vol. 34, no. 12 pp. 3271-3287, 2016.

[20] Arman Shojaeifard, Kai-Kit Wong, Khairi Ashour Hamdi, Emad Alsusa, Daniel K. C. So, Jie Tang, "Stochastic Geometric Analysis of EnergyEfficient Dense Cellular Networks," IEEE Access, vol. 5, pp. 455-469, 2017.

[21] Priyabrata Parida, Harpreet S. Dhillon, Pavan Nuggehalli, "Stochastic Geometry-Based Modeling and Analysis of Citizens Broadband Radio Service System,” IEEE Access, vol. 5, pp. 7326-7349, 2017.

[22] Min Wang, Hui Gao, Tiejun Lv, "Energy-Efficient User Association and Power Control in the Heterogeneous Network," Access IEEE, vol. 5, pp. 5059-5068, 2017.

[23] C. Xiong, G. Y. Li, S. Zhang, Y. Chen, and S. Xu,"Energy- and spectralefficiency tradeoff in downlink OFDMA networks," IEEE Trans. Wireless Commun., vol. 10, no. 11, pp. 38743886, Nov. 2011.

[24] Fereidoun H. Panahi and Tomoaki Ohtsuki,"Analytical Modeling of Cognitive Heterogeneous Cellular Networks over Nakagami-m Fading," IEEE Globecom 2014

[25] ElSawy, H., Hossain, E., and Haenggi, M. (2013). "Stochastic Geometry for Modeling, Analysis, and Design of Multi-Tier and Cognitive Cellular Wireless Networks: A Survey," IEEE Communications Surveys Tutorials, 15(3), 9961019.

[26] F. Baccelli, P. Miihlethaler, and B. Blaszczyszyn, "Stochastic analysis of spatial and opportunistic Aloha," IEEE J. Sel. Areas Commun., vol. 27, no. 7, pp. 11051119, Sep. 2009

[27] Chun-Wei Seah, Yew-Soon Ong, Ivor W. Tsang, Siwei Jiang, "Pareto Rank Learning in Multi-objective Evolutionary Algorithms," Evolutionary Computation (CEC) 2012 IEEE Congress on, pp. 1-8, 2012.

[28] Hui Li, Dario Landa-silva, Xavier Gandibleux, "Evolutionary multiobjective optimization algorithms with probabilistic representation based on pheromone trails," Evolutionary Computation (CEC) 2010 IEEE Congress on, pp. 1-8, 2010.

[29] J. G. Andrews, F. Baccelli, and R. K. Ganti, "A tractable approach to coverage and rate in cellular networks," IEEE Trans. Commun., vol. 59, no. 11, pp. 31223134, Nov. 2011.

[30] Zhi-Quan Luo and Wei Yu, "An introduction to convex optimization for communications and signal processing," IEEE Journal on Selected Areas in Communications, vol. 24 , no. 8 , Aug. 2006

[31] V. Rodriguez, "An analytical foundation for resource management in wireless communication,” in Proc. IEEE GLOBECOM, Dec. 15, 2003 vol. 2, pp. 898902

[32] A. Charnes, W.W. Cooper,"Goal programming and multiple objective optimization," part 1. Eur. J. Oper. Res. 1, pp.3954, 1977.

[33] J.S. Arora, O.A. Elwakeil, A.I. Chahande, C.C. Hsieh, "Global optimization methods for engineering applications: a review," Struct. Optim. 9, pp. 137159,1995

[34] T.W. Athan, P.Y.Papalambros,"A note on weighted criteria methods for compromise solutions in multi-objective optimization," Eng. Optim. 27, 155176, 1996

[35] H. Baier," ber Algorithmen zur Ermittlung und Charakterisierung Paretooptimaler Lsungen bei Entwurfsaufgaben Elastischer Tragwerke," Z Angew. Math. Mech. 57, 318320,1977.

[36] H. Kwon and T. Birdsall, "Channel capacity in bits per Joule," IEEE Journal of Oceanic Engineering, vol.11, no. 1, pp. 97-99, Jan. 1986.

[37] O. Somekh, B. M. Zaidel, and S. Shamai, "Sum rate characterization of joint multiple cell-site processing," IEEE Trans. Inf. Theory, vol. 53, no. 12, pp. 44734497, Dec. 2007.

[38] S. Verdu, "Spectral efficiency in the wideband regime," IEEE Trans. Inf. Theory, vol. 48, no. 6, pp. 13191343, June 2002.

[39] L. Deng, Y. Rui, P. Cheng, J. Zhang, Q.-T. Zhang, and M.-Q. Li, "A unified Energy Efficiency and Spectral Efficiency Tradeoff Metric in Wireless Networks," IEEE Communications Letters, vol. 17, no. 1, pp. 55-58, Jan. 2013.

[40] Hei YQ, Zhang C, Shi GM, "Trade-off optimization between energy efficiency and spectral efficiency in large scale MIMO systems," Energy, vol. 145, pp. 747-753 Feb. 2018.

[41] O. Onireti, F. Heliot, and M. A. Imran, "On the energy efficiency-spectral efficiency trade-off in the uplink of CoMP system," IEEE Trans. Wireless Commun., vol. 11, no. 2, pp. 556561, Feb. 2012.

[42] F. Heliot, M. A. Imran, and R. Tafazolli, "On the energy efficiency spectral-efficiency trade-off over the MIMO Rayleigh fading channel," IEEE Trans. Commun., vol. 60, no. 5, pp. 13451356, May 2012.

[43] C. Xiong, G. Y. Li, S. Zhang, Y. Chen, and S. Xu, "Energy- and spectral efficiency tradeoff in downlink OFDMA networks," IEEE Trans. Wireless Commun., vol. 10, no. 11, pp. 38743886, Nov. 2011.

[44] Chunlong He, Bin Sheng, Pengcheng Zhu, Xiaohu You,"Energy Efficiency and Spectral Efficiency Tradeoff in Downlink Distributed Antenna Systems," IEEE Wireless Communications Letters, vol. 1, no. 3, June 2012.

[45] O. Amin, E. Bedeer, M. Ahmed and O. Dobre, "Energy Efficiency- Spectral Efficiency Trade-off: A Multi-objective Optimization Approach," IEEE Transactions on Vehicular Technology, vol. 65, pp. 1975-1981, 2016.

[46] Z. Chen, L. Qiu, X. Wen Liang,"Area Spectral Efficiency Analysis of Multi-Antenna Two-Tier Cellular Networks," IEEE Communications Letters, vol. 21, no. 1, Jan. 2017.

[47] M. Lashgari, B. Maham, H. Kebriaei, "Energy-efficient self-backhauling in heterogeneous wireless networks: A game-theoretic approach," Physical Communication, vol. 29, pp. 296-306, August 2018

[48] X. Ge, B. Yang, J. Ye, G. Mao, C-X Wang, T. Han, "Spatial Spectrum and Energy Efficiency of Random Cellular Networks," IEEE Transactions on Communications, vol. 63, no. 3, pp. 1019 - 1030, March 2015

[49] L. Xiang, X. Ge, C-X Wang, F. Y. Li, F. Reichert,"Energy Efficiency Evaluation of Cellular Networks Based on Spatial Distributions of Traffic Load and Power Consumption," IEEE Transactions on Wireless Communications, vol. 12, no. 3, pp.961-973, March. 2013.

[50] X. Ge, H. Jia, Y. Zhong, Y. Xiao, Y. Li, B. Vucetic,"Energy Efficient Optimization of Wireless-powered 5G Full Duplex Cellular Networks: A Mean Field Game Approach," IEEE Transactions on Green Communications and Networking, vol. 3, no. 2, June 2019. 